\title{
Ischemia/reperfusion injury in coronary artery bypass grafting: Time to revisit?
}

\section{T. Bruce Ferguson, Jr, MD}

Surgical revascularization has demonstrated exceptional durability as a therapeutic intervention for ischemic heart disease. Objectively, major morbidity and mortality outcomes have improved steadily over the past 20 years, despite a well-documented increase in preoperative risk owing to cardiac and noncardiac comorbidity conditions. ${ }^{1}$ The overall risk-adjusted mortality for coronary artery bypass grafting (CABG) in 1989 exceeded 5\%; for 2007 to 2009 , the annual risk-adjusted mortality has hovered around 2.0\% nationwide according to data from The Society of Thoracic Surgeons national database. ${ }^{2}$ This remarkable improvement can be attributed to better preoperative patient selection, better intraoperative technical and perioperative management (anesthesia and perfusion technique improvements), and substantially improved perioperative care, both short term and long term.

It is of interest, then, that careful evaluation of these data from The Society of Thoracic Surgeons also documents that this risk-adjusted mortality for CABG has not continued to decline over the past 3 years, but rather has remained stable. Does this potentially suggest that the risk-adjusted mortality curve has reached its nadir, based on current technology and techniques, and in fact will start to increase as the preoperative risk continues to steadily increase?

Addressing this question may be more complicated than first appears. With this low overall risk-adjusted mortality, substantial improvement in this overall metric cannot be accomplished by improvement in the lowest-risk subset of this overall group. There simply are not enough patients in this subset with adverse events to affect the overall group metric; the persistent occurrence of random events in this lowestrisk subset is what David Eddy ${ }^{3}$ has characterized as "the probabilistic component" , medicine. ${ }^{3}$

It is rather the high-risk group of patients in this overall subset to which attention needs to be refocused to continue to drive down the overall operative mortality. Better understanding of the impact of pulmonary and/or renal insufficiency, concomitant hepatic disease, and the structural components of cardiac care on CABG morbidity and mortality are examples of investigations that are ongoing.

From the Department of Cardiovascular Sciences, East Carolina Heart Institute, The Brody School of Medicine at East Carolina University, Greenville, NC.

Received for publication April 1, 2010; accepted for publication April 10, 2010; available ahead of print May 24, 2010.

Address for reprints: T. Bruce Ferguson, Jr, MD, Chairman's Office, ECHI, 115 Heart Dr, Greenville, NC 27834 (E-mail: fergusont@ecu.edu).

J Thorac Cardiovasc Surg 2011;141:1-2

$0022-5223 / \$ 36.00$

Copyright $₫ 2011$ by The American Association for Thoracic Surgery doi:10.1016/j.jtcvs.2010.04.008
Additionally, new large-scale randomized trials are being designed in which conventional concepts of revascularization are being re-thought. In the ISCHEMIA and EXCEL trials, both in development, the interventional groups of cardiologists and surgeons have agreed to a conceptual definition of optimal revascularization that includes 2 components, namely, anatomic and ischemic revascularization. Up to now, the surgical approach has been largely anatomic, based on grafting of all coronaries with a pre-specified degree of proximal stenosis. As the concept of ischemic revascularization, where only areas with documented ischemia and significant epicardial arterial stenoses should be revascularized, takes root in percutaneous coronary interventions, there may be correlates to surgical revascularization that are documented through these trials to be quite important. In addition, new technologies are evolving for intraoperative assessment of both angiographic and functional (ischemic) components of surgical revascularization.,

This brings us back to ischemia/reperfusion. This is the focus of the methodologic considerations of the RED-CABG trial (Reduction in Cardiovascular Events by AcaDesine in Subjects Undergoing CABG), which, if completed, would be the largest randomized trial in the history of cardiac surgery. The hypothesis of the trial is that the acadesine, a first-in-class adenosine-regulating agent, will reduce the incidence of cardiac and noncardiac ischemia/reperfusion events in high-risk patients undergoing CABG. Previous trials assessing the use of acadesine in CABG have, in a 1997 meta-analysis, ${ }^{6}$ been associated with lower odds of perioperative myocardial infarction (MI) and a reduction in the composite incidence of cardiac death, acute MI, or stroke at 4 days postoperatively. The safety profile of acadesine in these trials documented only a transient hyperuricemia associated with drug administration. The results of these individual trials were not compelling enough to change clinical practice despite these promising results. However, these studies were performed early in the decade of the 1990s, when many of the cardiac and noncardiac confounding contributors to $\mathrm{CABG}$ mortality were more prevalent than they are today.

So is it time to revisit ischemia/reperfusion in CABG? The RED-CABG study outlines a substantial scientific rationale for the presence of ischemia/reperfusion in $\mathrm{CABG}$, related both to myocardial protection and to potential injurious effects from cardiopulmonary bypass. The RED-CABG trial is a randomized, double-blind, placebo-controlled, multinational clinical study of 7500 high-risk patients designed specifically to finally answer the question of ischemia/reperfusion in CABG. Although overall CABG mortality has declined, 
patients continued to die of either severe left ventricular dysfunction or, more commonly, other organ-system injury occurring at the time of surgery. Both of these circumstances would potentially be addressed by an amelioration of ische$\mathrm{mia} /$ reperfusion injury at the time of surgery.

The primary end point of all-cause mortality, nonfatal stroke, or need for mechanical support (intra-aortic balloon pump, ventricular assist device) for severe left ventricular dysfunction will be assessed in patients undergoing nonemergency $\mathrm{CABG}$ requiring cardiopulmonary bypass and cardioplegia who are 50 years of age or older, with one or more of the following risk factors: female gender, prior CABG, prior MI (except for MI less than 5 days before surgery), prior ischemic stroke, ejection fraction less than or equal to $30 \%$ on imaging within the previous 3 months, or diabetes mellitus requiring therapy. Patients must have significant coronary disease on angiography within the past 12 months, including $50 \%$ or greater stenosis of the left main coronary artery, $70 \%$ or greater stenosis of 3 coronary arteries, or $70 \%$ or greater stenosis of 2 coronary arteries, one of which must be the left anterior descending coronary artery. Patients with MI greater than 2 years old lose the prior MI characteristic as a risk factor. The study is confined to patients undergoing CABG surgery without valve surgery, except for mitral valve repair. There are a number of additional exclusion criteria outlined in the article. The study uses a 1:1 randomized treatment assignment within 24 hours before the scheduled operation, stratified by gender. Patients are randomized to receive placebo intravenous infusion beginning 30 minutes before anesthesia and continuing for 7 hours postoperatively or the acadesine intravenous infusion over the same time frame. Follow-up at 28 days for the primary end point and by telephone at 6 months for survival is proscribed. Although robust, this protocol is very adaptable and applicable for most high-risk patients undergoing CABG with cardiopulmonary bypass. As scheduled, 2 futility analyses will be performed, at 2250 and then and again at 3000 patients enrolled.

Importantly, the mechanism of action of acadesine at the cellular level is still unknown; however, it remains the leading candidate adenosine-regulating agent, has a safety profile already validated in the setting of $\mathrm{CABG}$, and has potential applicability to other areas of ischemia/reperfusion if RED-CABG yields a positive result. This includes, for example, potential applicability in the complex setting of acute coronary syndrome.

Finally, RED-CABG is a critically important trial for the cardiac surgical community. As was the case with the PREVENT IV trial, ${ }^{7}$ RED-CABG is likely to yield significant new and important information regarding contemporary surgical revascularization. The potential to positively affect CABG outcomes by amelioration of ischemia/reperfusion is more likely to be demonstrable today as opposed to 20 years ago, for the reasons outlined above; this circumstance also makes it more likely that pharmacologic intervention, rather than technical issues or processes of care, will generate a positive impact. Importantly, the setting of cardiac surgery, represented here by CABG, has not been highly evaluated or used for pharmacologic intervention evaluations, and thus RED-CABG represents an important opportunity for this reason.

Amelioration of ischemia/reperfusion may be the necessary next step in the cardiac surgeon's continuing efforts to drive down perioperative mortality and morbidity in contemporary CABG. The entire cardiac surgery community eagerly awaits the results of the RED-CABG trial intervention.

\section{References}

1. Ferguson TB, Jammill BD, DeLong EL, Peterson ED, Grover FL. A decade of change: risk profiles and outcomes for isolated CABG procedures, 1990-1999. Ann Thorac Surg. 2002;73:480-90.

2. STS National Database Executive Summary 4th Q 2009 Data. Accessed on March 31, 2010 at http://www.sts.org/documents/pdf/ndb2010/20094thHarvestExecutive Summary.pdf.

3. Eddy DM. Benefit language: criteria that will improve quality while reducing costs. JAMA. 1996;275:650-7.

4. Leacche M, Balaguer JM, Byrne JG. Intraoperative grafts assessment. Semin Thorac Cardiovasc Surg. 2009;21:207-12.

5. Desai ND, Miwa S, Kodama D, Koyama T, Cohen G, Pelletier MP, et al. A randomized comparison of intraoperative indocyanine green angiography and transit-time flow measurement to detect technical errors in coronary bypass grafts. J Thorac Cardiovasc Surg. 2006;132:585-94.

6. Mangano DT. Effects of acadesine on myocardial infarction, stroke, and death following surgery. A meta-analysis of the 5 international randomized trials. The Multicenter Study of Perioperative Ischemia (McSPI) Research Group. JAMA. 1997;277:325-32.

7. Alexander JH, Hafley G, Harrington RA, Peterson ED, Ferguson TB Jr, Lorenz TJ, et al; PREVENT IV Investigators. Efficacy and safety of edifoligide, an E2F transcription factor decoy, for prevention of vein graft failure following coronary artery bypass graft surgery: PREVENT IV: a randomized controlled trial. JAMA. 2005; 294:2446-54. 